Questo sito o gli strumenti terzi da questo utilizzati si avvalgono di cookie necessari al funzionamento ed utili alle finalit illustrate nella cookie policy. Se vuoi saperne di pi o negare il consenso a tutti o ad alcuni cookie, consulta la cookie policy.

Chiudendo questo banner, scorrendo questa pagina, cliccando su un link o proseguendo la navigazione in altra maniera, acconsenti all uso dei cookie. Ho capito Cookies Policy Home Page Chi siamo Collane E-book Riviste Journals 9 Come acquistare Book orders 1 Come contattarci Help Login/l tuoi dati Password? $\begin{gathered}17.000 \text { volumi, } 900 \text { novità, } \\ \text { oltre } 80 \text { periodici, } \\ \text { più di } 30.000 \text { autori... } \\ \begin{array}{r}\text { dal } 1955 \\ \text { catalogo }\end{array}\end{gathered}$
$\begin{gathered}\text { il più grande } \\ \text { specializzato in Italia }\end{gathered}$

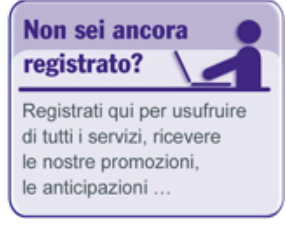

\begin{tabular}{|l}
\hline $\begin{array}{l}\text { Elenco alfabetico delle } \\
\text { riviste }\end{array}$ \\
\hline $\begin{array}{l}\text { Riviste (in corso) } \\
\text { per disciplina }\end{array}$ \\
\hline $\begin{array}{l}\text { Come effettuare } \\
\text { una ricerca }\end{array}$ \\
\hline Servizi per gli Autori \\
\hline $\begin{array}{l}\text { Servizi per biblioteche } \\
\text { ed enti }\end{array}$ \\
\hline
\end{tabular}
ed enti

Come abbonarsi alla versione cartacea

Come abbonarsi alla versione online

\section{Servizi online}

per atenei

Servizi

per utenti privati

Abbonarsi ad una versione cartacea o online

Scaricare un articolo online

II costo di un download credit

Acquistare un download credit

Acquistare un singolo fascicolo come e-book

2.4 Foreign orders

\section{Come acquistare} arretrati Canoni abbonamenti

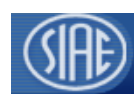

Fotocopie e diritto d'autore: domande e risposte

C CleARedi

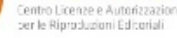

Fare copie "in regola" è più semplice di quanto si possa pensare...
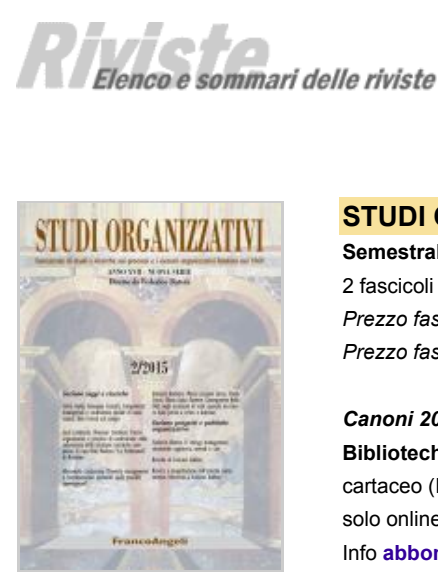

\section{STUDI ORGANIZZATIVI}

Semestrale di studi e ricerche sui processi e i sistemi organizzativi

2 fascicoli all'anno, ISSN 0391-8769, ISSNe 1972-4969

Prezzo fascicolo (inclusi arretrati): $€ 35,50$

Prezzo fascicolo e-book (inclusi arretrati): $€ 30,00$

\section{Canoni 2016}

Biblioteche, Enti, Società:

cartaceo (IVA inclusa): Italia € 70,50; Estero $€ 109,00$

solo online (IVA esclusa): $€ 78,00$

Info abbonamenti cartaceo Info licenze online

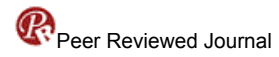

Atenei:

online (con arretrati, accesso perpetuo, formula plus )

Info licenze online

Privati:

cartaceo Italia $€ 62,50$; cartaceo Estero $€ 95,50$; solo online (privati) $€ 53,00$ Info abbonamenti Info Soci AIS

Proporre un articolo

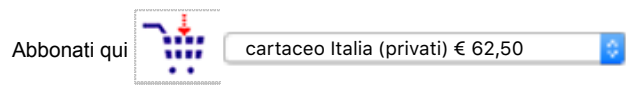

\begin{tabular}{l|l|l|l|l||l|} 
Progetto e fini & Direzione editoriale & Contatti & Referee & Ranking & Indicizzazione \\
\hline
\end{tabular}

\begin{tabular}{l|l|l||l} 
Norme redazionali & Codice Etico & Memberships & Audience e diffusione \\
\hline
\end{tabular}

Fitt Articolo gratuito

르 Articolo a pagamento

ᄀำ.ำ Fascicolo disponibile in e-book

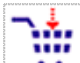
$\because:$ Acquista il fascicolo in formato e-book

Fascicolo 2/2014

Fite Federica Persico, La gestione di un evento estremo come processo dinamico di coordinamento organizzativo

Mara Brumana, Maurizio Decastri, Danila Scarozza, Stefano Za, Innovazione tecnologica e organizzazione:

E trend, aree di ricerca e prospettive

- Raimondo Ingrassia, Accountability, organizzazione e rendicontazione sociale nelle amministrazioni giudiziarie

ZZ italiane: alcune evidenze empiriche

7 Mario D'Andreta, Le basi culturali del policy-making: struttura e discorso dell'élite di Asti sullo sviluppo locale

- Lorenzo Ciapetti, Paolo Perulli, Open Manufacturing: una piattaforma interregionale per l'innovazione nel

Z settore della meccatronica. Nuove evidenze per una nuova stagione di politiche regionali dell'innovazione

Federico Butera, Futuro professionale: dal taylor- fordismo ai nuovi modi di produzione. I frantumi ricomposti,

즈 1971-20151

Fiet Notizie sugli autori

ᄀ:⿱艹:犬:

Acquista il fascicolo in formato e-book Fascicolo $1 / 2014$

Attila Bruni, Laura Lucia Parolin, Dalla produzione automatizzata agli ambienti tecnologicamente densi: la

Fitet Attila Bruni, Laura Lucia Parolin, Dalla produzione

Enrico Maria Piras, Alberto Zanutto, "Le infermiere sanno quello che i medici vogliono": il lavoro di

Z2 congiunzione nei TDEs

- Stefano Crabu, Allineare umani, tecnologie e saperi: il lavoro infrastrutturante negli ambienti tecnologicamente

르 densi

ZZ Roberto Cibin, Lorenzo Ruzzene, Francesca Gleria, L'apertura dei dati pubblici nella PA: il progetto Open Data
Sottoscrivi il FEED

per conoscere subit

ultimo fascicolo di questa Rivista

English version

LE RIVISTE

DEL MESE

Q

\begin{tabular}{|l|}
\hline iconomia \\
\hline Aanagement \\
\hline 'sicologia e Psicoterapia \\
\hline iociologia \\
\hline icienze del territorio \\
\hline 'olitica, diritto \\
\hline icienze della formazione \\
\hline itoria \\
\hline iilosofia \\
\hline
\end{tabular}

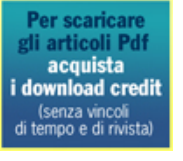

Suggerite alla Vostra Biblioteca di abbonarsi

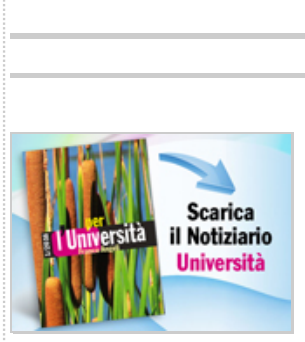




\section{in Trentino}

7 Barbara Mellini, Sabina Giorgi, Alessandra Talamo, Soglie, scene, spazi e artefatti come strumenti di

즈 conoscenza dell'organizzazione

F Federico Butera, Note sulla storia dell'automazione. Dall'impatto sociale dell'automazione alla progettazione

Z congiunta di tecnologia, organizzazione e sviluppo delle persone

- Maurizio Teli, Vincenzo D'Andrea, Antonella De Angeli, La progettazione partecipata come pratica e politica per

Z_ costruire ambienti tecnologicamente densi

- Francesca Alby, Cognizione-in-interazione, azione pratica e artefatti: il contributo della ricerca psicologica allo

ZL studio della relazione fra tecnologie e comunità lavorative

Fit. Notizie sugli autori

\section{Sommari delle annate}

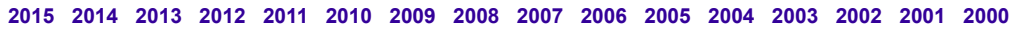
1999

FrancoAngeli è membro della Publishers International Linking Association, Inc (PILA) associazione indipendente e no profit per facilitare (attraverso i servizi tecnologici implementati da CrossRef.org) l'accesso degli studiosi ai contenuti digitali nelle pubblicazioni professionali e scientifiche.

\section{A FrancoAngeli}




\title{
Innovazione tecnologica e organizzazione: trend, aree di ricerca e prospettive
}

\author{
di Mara Brumana, Maurizio Decastri, Danila Scarozza, \\ Stefano Za*
}

\section{Executive summary}

Negli ultimi anni, l'innovazione tecnologica è stata una delle tematiche più dibattute nella letteratura accademica, tuttavia le sue implicazioni per le realtà organizzative, così come per gli stessi attori organizzativi sembrano ancora non sufficientemente teorizzate ed empiricamente analizzate. Dopo l'attenzione dedicata alla tecnologia negli studi organizzativi intorno agli anni' 50 del secolo scorso si è assistito, infatti, a un declino nell'interesse verso questo tema, accentuatosi ancor di più negli ultimi due decenni. L'obiettivo di questo articolo, pertanto, è quello di contribuire al dibattito su innovazione tecnologica, organizzazione e lavoro.

Muovendo da queste premesse, è stata condotta una ricerca sulla piattaforma ISI Web of Science utilizzando la chiave di ricerca "technolog* innovation*". Nel periodo 1985-2013 sono stati identificati 1028 contributi scientifici pubblicati su 89 riviste internazionali - selezionate combinando 3 gruppi di riviste diversi, ma complementari quali il Financial Times ranking, riviste di matrice sociologica e il Technology Innovation Management Journals ranking. Un'analisi bibliometrica basata sull'utilizzo di indicatori descrittivi e di strumenti di Social Network Analysis, ha poi consentito di analizzare i 1028 contributi scientifici raccolti. In particolare, è stato analizzato l'andamento (in termini di numerosità) delle pubblicazioni e l'impatto che i contributi selezionati hanno avuto sulla comunità scientifica di riferimento (trend delle citazioni). Altre analisi hanno poi consentito di identificare gli autori più produttivi, la loro distribuzione geografica (in base alle università di appartenenza) e le principali co-authorship. Infine, un'analisi approfondita è stata condotta sui topic principalmente trattati dal dataset selezionato.

All'analisi del dibattito internazionale si è affiancato un approfondimento sul sottoinsieme dei contributi italiani, ovvero quelli pubblicati da almeno un autore affiliato in una università italiana. Si sono così potute evidenziare, utilizzando le

\footnotetext{
* Gli autori sono riportati in ordine alfabetico.

Studi organizzativi n. 2, 2014 - Sezione saggi e ricerche
} 
stesse analisi, le peculiarità della realtà del nostro paese per quanto riguarda la ricerca sull'innovazione tecnologica.

I risultati ottenuti sia a livello internazionale che italiano, rivelano, nel corso del periodo considerato, una crescita significativa della letteratura su questo tema e una crescente attenzione allo stesso, la multidisciplinarietà della tematica osservata, la rilevanza dei contributi di management e business \& economics, ma soprattutto la necessità e allo stesso tempo opportunità di investigare il ruolo dell'innovazione tecnologica nell'ambito organizzativo. Dall'analisi condotta sui contributi italiani, inoltre, emerge che l'innovazione tecnologica è maggiormente correlata a tematiche economiche-sociali evidenziando ancora la scarsa attenzione prestata al legame tra innovazione tecnologica, organizzazione e lavoro.

Keywords:Innovazione tecnologica, organizzazione, lavoro, analisi bibliometrica, Social Network Analysis, ISI Web of Science

\begin{abstract}
Over the years theoretical perspectives and empirical research on technological innovation have developed together with theoretical and empirical studies on organization. However, after a great deal of attention devoted to technology in organization studies in the 50s of the last century, there has been a decreasing interest about this topic. Aiming to contribute to the debate on technological innovation, organization and work, a search in the ISI Web of Science platform was performed using the keyword "technolog* innovation*" and looking at 89 journals identified from three complementary groups of journals. To analyze the 1028 scientific retrieved contributions, published between 1985 and 2013, a bibliometric analysis has been conducted, adopting also Social Network Analysis tools. The analysis on the whole dataset was supplemented by the study of the subset of the Italian contributions - i.e. those in which at least one author was affiliated with an Italian university - in order to point out the peculiarities of the research on technological innovation conducted by Italian scholars. The results obtained for both the whole sample and the Italian one reveal a significant growth of the technological innovation literature over the investigated period, the multidisciplinarity of the field, the relevance of management and business \& economics contributions, but in particular the necessity and opportunity to investigate the role of technological innovation within the organizational life.
\end{abstract}

Keywords: Technological innovation, organization, work, bibliometric analysis, Social Network Analysis, ISI Web of Science. 


\section{Innovazione tecnologica, organizzazione e lavoro: un inqua- dramento teorico}

Nel corso degli anni ricerche e prospettive di analisi sull'innovazione tecnologica si sono sviluppate parallelamente a studi e prospettive teoriche sull' organizzazione (Orlikowski, 2000). Inoltre, cospicui sono stati i cambiamenti che hanno condotto al ripensamento di ruoli e funzioni che tanto la tecnologia, quanto l'organizzazione assolvono sia all'interno del dibattito teorico e scientifico, sia a livello "pratico". É proprio in risposta ai suddetti cambiamenti che studiosi e ricercatori hanno iniziato ad analizzare le implicazioni dell'innovazione tecnologica in chiave organizzativa (Cook e Brown, 1999; Tushman, M.; Anderson e O’Reilly, 1997).

L'innovazione può essere definita come la produzione o adozione, assimilazione e utilizzo di un'idea o di un comportamento che è nuovo per l'organizzazione. Essa può consistere in un nuovo prodotto, un nuovo servizio, un nuovo processo, un nuovo modello di business, una nuova tecnologia, o una nuova pratica amministrativa (Crossan e Apaydin, 2010; Hage, 1999). Con specifico riferimento all'innovazione tecnologica, alcuni autori la definiscono come la generazione di nuovi prodotti e processi, o di significativi miglioramenti tecnologici in prodotti e processi esistenti (Becheikh, Landry e Amara, 2006; Galende, 2006). In particolare, quando l'innovazione tecnologica non interessa esclusivamente i prodotti, bensì i processi, questa implica cambiamenti nelle strutture organizzative, nell'organizzazione del lavoro, ma anche cambiamenti nelle tecniche e negli strumenti di lavoro. Questi ultimi, a loro volta, generano cambiamenti nelle competenze richieste e nei ruoli professionali (Davies, Dawson e Francis, 1973; Meissner, 1970).

Date queste premesse, e a prescindere dall'ampiezza e dall'esaustività della definizione adottata, l'importanza che l'innovazione tecnologica riveste per le imprese che competono in un ambiente in continua evoluzione sembra, considerati gli scenari attuali, ormai innegabile. Appare esserci consenso, sia nel mondo accademico che in quello del business, sul fatto che la conoscenza tecnologica e la capacità innovativa risultano essere delle risorse chiave per le imprese, nonché uno dei principali fattori determinanti le loro performance (De Massis, Frattini e Lichtenthaler, 2013; Crossan e Apaydin, 2010; Becheikh et al., 2006; Galende, 2006; Prajogo e Ahmed, 2006). La rilevanza dell'innovazione tecnologica è legata anche al suo impatto sulla sfera economica e sociale, sia a livello macro - occupazione, struttura dei settori, composizione della domanda di lavoro, posizione com- 
petitiva delle nazioni, crescita economica, benessere della società - che a livello micro - competitività del business e definizione delle strategie (Crossan e Apaydin, 2010; Nieto, 2003).

Tuttavia, come già notato da alcuni autori (Mansell, Avgerou, Quah e Silverstone, 2007; Zammuto, Griffith, Majchrzak, Dougherty e Faraj, 2007; Dewett e Jones, 2001), sebbene il processo di innovazione tecnologica e i suoi risultati (Crossan e Apaydin, 2010; Garud, Tuertscher e Van de Ven, 2013) pervadano sempre più l'impresa moderna, il legame tra tecnologia, organizzazione e lavoro appare non ancora sufficientemente teorizzato ed empiricamente analizzato. La necessità di investigare il rapporto tra tecnologia, organizzazione e lavoro è confermata anche dalle più recenti rassegne della letteratura (Orlikowski e Scott, 2008; Zammuto et al., 2007). Le risultanze di queste review mostrano, infatti, che dopo una grande attenzione dedicata alla tecnologia negli studi organizzativi intorno agli anni '50 del secolo scorso, si è assistito a un declino nell'interesse e nell'attenzione verso questo tema, accentuatosi ancor di più negli ultimi due decenni. In particolare, come riportato da Orlikowski e Scott (2008), da gennaio 1997 a dicembre 2006 più del 95\% degli articoli pubblicati nei cosiddetti top management journals non prende in considerazione il ruolo e l'impatto della tecnologia nella vita organizzativa. La maggior parte della letteratura economica e manageriale, da Schumpeter in poi $(1934$; 1942), sembra infatti essersi prevalentemente focalizzata sulle determinanti dell'innovazione (Crossan e Apaydin, 2010; Ahuja, Lampert e Tandon, 2008; Becheikh et al., 2006) trascurando gli effetti e le influenze che questa inevitabilmente esercita tanto sui processi organizzativi, quanto sulle persone. Questa disattenzione risulta essere problematica soprattutto se si considera che il ricorso a nuove tecnologie: a) modifica sia gli aspetti strutturali che quelli sociali all'interno dell'organizzazione; $b$ ) media e influenza le attività tra imprese, industrie ed economie (Zammuto et al., 2007).

Questo articolo si colloca all'interno di una ricerca più ampia volta a comprendere se e come l'innovazione tecnologica influenza i processi produttivi delle organizzazioni in settori produttivi diversi, modificandone assetti, ruoli, processi decisionali, sistemi e logiche di gestione delle risorse umane. In particolare, la rassegna della letteratura presentata in questo articolo si inserisce nel dibattito menzionato con un duplice scopo:

a) da un lato, l'obiettivo è quello di offrire una panoramica sulla letteratura organizzativa $e$ non avente ad oggetto l'innovazione tecnologica in questi ultimi 30 anni al fine di evidenziare come il significato e l'importanza di tale concetto si siano evoluti. 
b) dall'altro provare a identificare e classificare (sia nel tempo, sia concettualmente) aree di studio e di ricerca (anche futura) su tecnologia, organizzazione e lavoro.

All'analisi del quadro d'insieme si affianca un approfondimento su quanto fatto da studiosi e ricercatori italiani. L'obiettivo è quello di mettere in evidenza le peculiarità del contesto nazionale nella ricerca sull'innovazione tecnologica e i suoi possibili impatti sull'organizzazione e sul lavoro.

Rispetto alle review della letteratura esistenti, la presente analisi si distingue per almeno tre motivi: (i) il set di riviste internazionali considerato comprende journal manageriali e di business, riviste di matrice sociologica e riviste specificamente dedicate al tema dell'innovazione tecnologica; (ii) vengono utilizzati strumenti di analisi bibliometrica e in particolare di Social Network Analysis (SNA); (iii) viene proposto un approfondimento sulla realtà italiana.

\section{Metodologia}

Da un punto di vista metodologico e al fine di perseguire gli obiettivi sopra esposti, l'analisi della letteratura si è articolata in tre fasi principali: (i) identificazione delle riviste internazionali su cui effettuare l'analisi; (ii) scelta delle parole chiave da utilizzare per la ricerca di contributi scientifici nel set di riviste individuato; (iii) analisi bibliometrica delle pubblicazioni selezionate secondo i criteri precedentemente definiti, utilizzando anche gli strumenti della SNA.

Relativamente al primo punto e in linea con gli obiettivi di questo contributo, l'attenzione non è stata limitata ai soli top journal di management (e business) - così come fatto da precedenti review della letteratura (Orlikowski e Scott, 2008; Zammuto et al., 2007). Sono stati, infatti, considerati 3 gruppi di riviste diversi ma complementari, per un totale di 96 riviste così distribuite:

- 45 riviste del ranking del Financial Times (FT45) ${ }^{1}$ : si tratta di un rating la cui rilevanza è ampiamente riconosciuta nel mondo accademico e in particolare dagli studiosi di management (Burgess e Shaw, 2010) (tabella 1);

\footnotetext{
${ }^{1}$ http://www.ft.com/intl/cms/s/2/3405a512-5cbb11e18f1 f00144feabdc0.html\#axzz2YYYxf4nd
} 
- 36 riviste di matrice sociologica ${ }^{2}$ selezionate sia tra i top journal individuati dall'Agenzia Nazionale di Valutazione del Sistema Universitario e della Ricerca (ANVUR) nell'Area "Scienze politiche e sociali", sia tra le riviste di alcuni ranking internazionali relativi alle aree "sociology", "social work" e, più in generale, "social sciences" (si vedano al riguardo Jacobs (2001); il Journal Citation Reports Social Sciences Edition pubblicato da Thomson Reuters $(2012)^{3}$; lo SCImago Journal \& Country Rank (2012) ${ }^{4}$ ) (tabella 2);

- 15 riviste del ranking 'Technology Innovation Management Journals' (TIM): si tratta di un rating identificato da Thongpapanl e pubblicato, nella sua ultima versione, sulla rivista Technovation (2012). Questo ranking ha attratto particolare interesse e attenzione, soprattutto negli ultimi due decenni (Cheng, Kumar, Motwani, Reisman e Madan, 1999; Liker, 1996; Linton e Thongpapanl, 2004) (tabella 3).

Come già fatto da altri autori (per esempio Knoben e Oerlemans, 2006), è stata utilizzata la piattaforma ISI (Institute for Scientific Information) Web of Science (ISI-WoS) ${ }^{5}$ per la ricerca delle pubblicazioni, dopo aver verificato la presenza di tutte le riviste selezionate (7 riviste del secondo gruppo sono state escluse in quanto non presenti su ISI Web of Science) $)^{6}$.

Tabella 1 - Riviste Financial Times(FT45) ranking

\begin{tabular}{cl}
\hline$N$. & Rivista \\
\hline 1 & Academy of Management Journal \\
2 & Academy of Management Perspectives \\
3 & Academy of Management Review \\
\hline
\end{tabular}

${ }^{2}$ Questo secondo gruppo di riviste comprende anche 'Organization Studies' e 'Industry and Innovation', che non sono state considerate perché ricomprese, rispettivamente nel 'Financial Times' e nel 'Technology Innovation Management Journals' ranking.

${ }^{3} \mathrm{http}: / /$ ip-science.thomsonreuters.com/cgi-bin/jrnlst/jlresults.cgi? $\mathrm{PC}=\mathrm{SS}$

${ }^{4} \mathrm{http}: / /$ www.scimagojr.com/journalrank.php? category $=3323 \&$ area $=3300 \&$ year $=2012 \&$ coun try $=\&$ order $=$ sjr\&min $=0 \& \min$ type $=$ cd\&page $=0$

Nello specifico, all'interno della subject area 'social sciences', i journal sono stati individuati all'interno delle seguenti subject category: 'social work', 'social sciences' e 'sociology and political sciences'

${ }^{5} \mathrm{http}: / /$ apps.webofknowledge.com

${ }^{6}$ International Journal of Sociology; Journal of The Society for Social Work and Research; Review of Sociology; Science, Technology and Human Values; Science and Society; Sociological Practice; The American Journal of Sociology. 


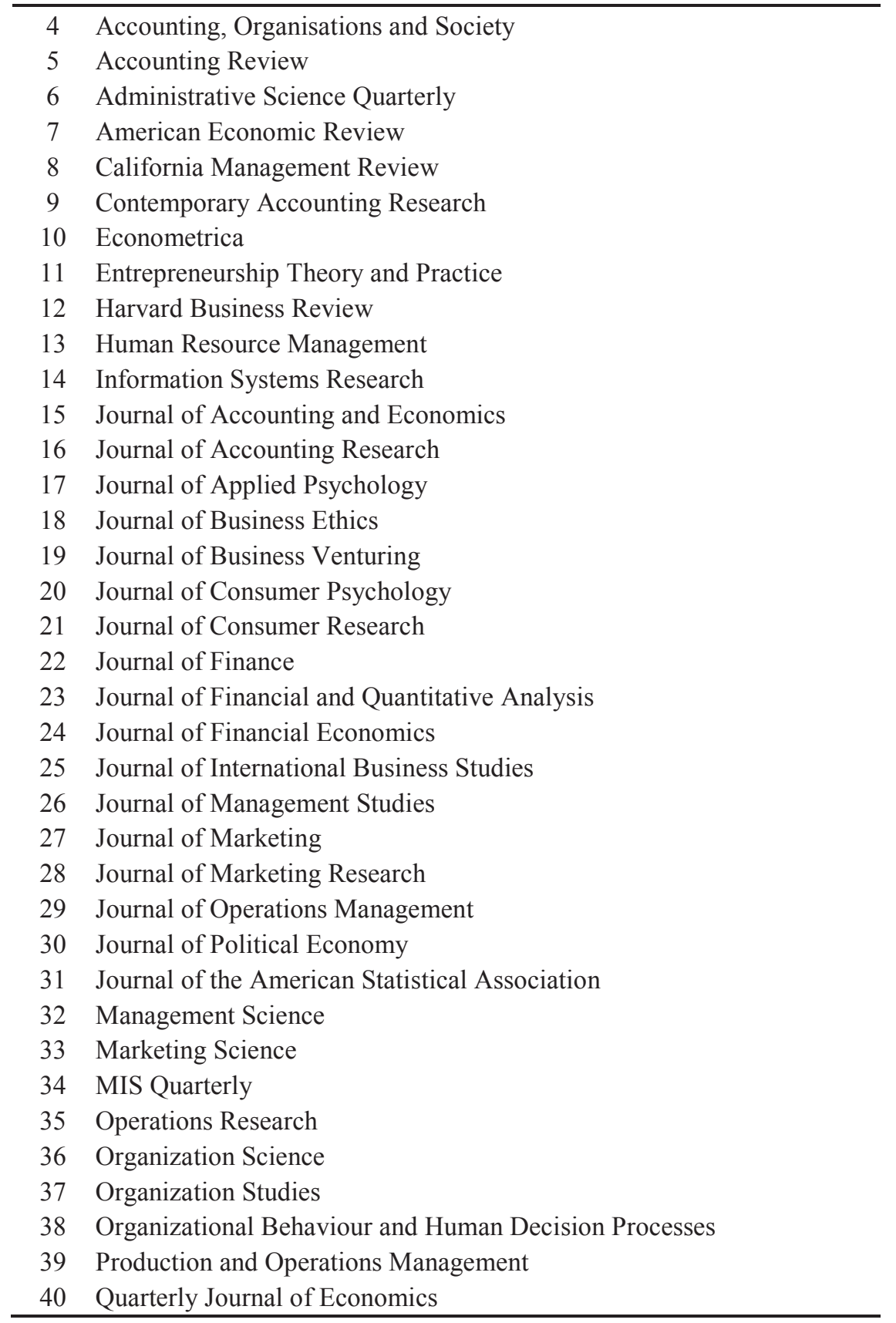




\begin{tabular}{ll}
\hline 41 & Rand Journal of Economics \\
42 & Review of Accounting Studies \\
43 & Review of Financial Studies \\
44 & Sloan Management Review \\
45 & Strategic Management Journal \\
\hline
\end{tabular}

Tabella 2 - Riviste aree Sociology, Social Work e Social Sciences

\begin{tabular}{cl}
\hline$N$. & Rivista \\
\hline 1 & Administration in Social Work \\
2 & American Sociological Review \\
3 & Annual Review of Sociology \\
4 & British Journal Of Industrial Relations \\
5 & British Journal of Sociology \\
6 & European Journal of Industrial Relations \\
7 & European Journal of Social Work \\
8 & European Sociological Review \\
9 & Human Relations \\
10 & Industrial Relations \\
11 & Industry \& Innovation \\
12 & Journal of Social Work Practice \\
13 & International Journal of Social Welfare \\
14 & International Journal of Sociology \\
15 & International Social Work \\
16 & Journal of Organizational Change Management \\
17 & Journal of Social Issues \\
18 & Journal of Social Work \\
19 & Journal of The Society for Social Work and Research \\
20 & Journal of Sociology \\
21 & Organization \\
22 & Qualitative Social Work \\
23 & Research on Social Work Practice \\
24 & Review of Sociology \\
25 & Scandinavian Journal of Management \\
26 & Science, Technology and Human Values \\
27 & Science and Society \\
28 & Social Problems \\
29 & Sociological Perspectives \\
30 & Sociological Practice \\
\hline &
\end{tabular}




\begin{tabular}{ll}
\hline 31 & Sociological Quarterly \\
32 & Sociological Review \\
33 & Sociological Theory \\
34 & Sociology \\
35 & The American Journal of Sociology \\
36 & Work Employment and Society \\
37 & Work and Occupations \\
\hline
\end{tabular}

Tabella 3 - Riviste Technology Innovation Management Journals (TIM) ranking

\begin{tabular}{cl}
\hline$N$. & Rivista \\
\hline 1 & Journal of Product Innovation Management \\
2 & Research Policy \\
3 & Research-Technology Management \\
4 & R\&D Management \\
5 & IEEE Transaction Engineering Management \\
6 & Technological Forecasting and SocialChange \\
7 & International Journal of Technology Management \\
8 & Technovation \\
9 & Technology Analysis and Strategic Management \\
10 & Journal of Engineering and Technology Management \\
11 & Engineering Management Journal \\
12 & Journal of Technology Transfer \\
13 & Science and Public Policy \\
14 & Industrial and Corporate Change \\
15 & Industry and Innovation \\
\hline
\end{tabular}

Per quanto riguarda la keyword per la ricerca, dopo diverse prove eseguite per identificare quella più utile allo scopo, si è deciso di utilizzare come unica parola chiave "technolog* innovation" da cercare come "TO$P I C$ " di ogni contributo. In ISI-WoS, le parole specificate nel campo "TOPIC" sono cercate nel titolo, nell'abstract e nelle keyword di ogni pubblicazione. L'asterisco nella keyword indica zero o più caratteri (ad esempio technology, technologies or technological). La ricerca della keyword identificata nelle 89 riviste dal 1985 a oggi $^{7}$ ha restituito 1028 risultati.

${ }^{7}$ Il 1985 è stato scelto come anno di partenza poiché la copertura cronologica del database ISI risale a questo anno. La ricerca è stata effettuata per l'ultima volta in data 7 agosto 2013. 
Con riferimento al terzo punto, infine, è stata condotta un'analisi bibliometrica sui 1028 contributi rilevati, utilizzando anche gli strumenti di Social Network Analysis (SNA). Di recente, infatti, l'utilizzo di strumenti di SNA applicati all'analisi della letteratura, soprattutto nell'ambito delle scienze sociali, risulta avere una discreta diffusione (Chabowski, Samiee e Hult, 2013; Vogel, 2012). Questo tipo di analisi consiste nell'applicazione di metodi statistici per esaminare il comportamento di una comunità scientifica (o più comunità) in base ai dati delle relative pubblicazioni (Leydesdorff, 2001).

\section{Analisi e risultati}

Nel periodo osservato, sono stati pubblicati 1028 contributi scientifici, di cui $829(81 \%)$ articoli, $44(4 \%)$ proceeding papers, $126(12 \%)$ review e altre recensioni non solo di librie $26(3 \%)$ editoriali, (il dataset include anche una nota, una correzione e un meeting abstract). Tutte le tipologie di documento sono state mantenute nel dataset sia perché l'obiettivo della presente ricerca è quello di fornire una panoramica complessiva della letteratura sull'innovazione tecnologica, sia perché tutti questi documenti danno, anche se in misura non eguale, un contributo sostanziale a tale letteratura.

Per quanto riguarda la distribuzione delle 1028 pubblicazioni nelle riviste selezionate, il 76\% (778) è stato pubblicato su journal appartenenti al TIM ranking - in particolare, 144 (14\%) appaiono su Research Policy, 121 (12\%) su Technovation, 101 (10\%) sull'International Journal of Technology Management - il 21\% (220), invece, è stato pubblicato nelle riviste del FT45 ranking - per esempio, 29 (3\%) sono state pubblicate su Organization Science, 26 (2,6\%) su Strategic Management Journal e 15 (1,5\%) su Management Science. Solo il 3\% (30) risulta essere pubblicato sulle riviste di matrice sociologica - in particolare, $5(0,5 \%)$ sono le pubblicazioni su Human Relations e altrettante $(0,5 \%)$ sul Journal of Organizational Change Management.

L'analisi bibliometrica delle 1028 pubblicazioni si è articolata in: (i) un'analisi di alcuni indicatori descrittivi, quali l'evoluzione del numero di pubblicazioni e citazioni per anno, la distribuzione dei contributi per università di appartenenza degli autori, la distribuzione dei contributi per Stato in cui è collocata tale università, (ii) l'utilizzo di strumenti di SNA con l'obiettivo di identificare alcune indicazioni interessanti circa la produttività degli autori e le eventuali relazioni di collaborazione tra essi, ma soprat- 
tutto circa il contenuto dei contributi selezionati. A tal fine è stata condotta un'analisi della loro distribuzione rispetto sia alle Web of Science subject categories, che alle Web of Science research areas, nonché un'analisi delle keywords presenti in ciascun contributo.

Anche l'approfondimento sui contributi italiani, ovvero quelli pubblicati da almeno un autore affiliato in una università italiana (56 pubblicazioni) ha visto il succedersi di due momenti di analisi - indicatori descrittivi e applicazione degli strumenti di SNA.

\subsection{Analisi descrittiva}

La figura 1 riporta il numero di pubblicazioni per anno dal 1985 al 2013. Come mostrato dal grafico, il numero di pubblicazioni è cresciuto negli anni e, in particolare, nel periodo 2006-2012 (fino a raggiungere 89 pubblicazioni nel 2012).

Più in generale, possono essere identificate tre fasi:

- fase "emergente" (dal 1985 al 1991): questo primo periodo è caratterizzato da una produzione moderata (dai 4 ai 10 contributi per anno);

- fase di "interesse crescente" (dal 1992 al 2005): in media, il numero di pubblicazioni è maggiore rispetto al periodo precedente e piuttosto costante nei vari anni (nello specifico raddoppia passando dai 7 contributi pubblicati in media nel primo periodo ai 13 pubblicati in questa seconda fase);

- fase di "rafforzamento" (dal 2006 al 2012): dopo il 2005, il numero di pubblicazioni è caratterizzato da un balzo verso l'alto. La crescita consistente dei contributi durante questa fase (con una media annua pari a circa 30 pubblicazioni) mostra il forte e consolidato interesse della comunità scientifica verso questo argomento.

I dati ottenuti per l'anno 2013 sono da considerarsi parziali poiché la ricerca è stata condotta in data 7 agosto 2013.

L'andamento del numero di pubblicazioni per anno all'interno del dataset dei 1028 contributi identificati è stato poi confrontato con il trend complessivo delle pubblicazioni nelle 89 riviste considerate. 
Figura 1 - Numero di pubblicazioni per anno

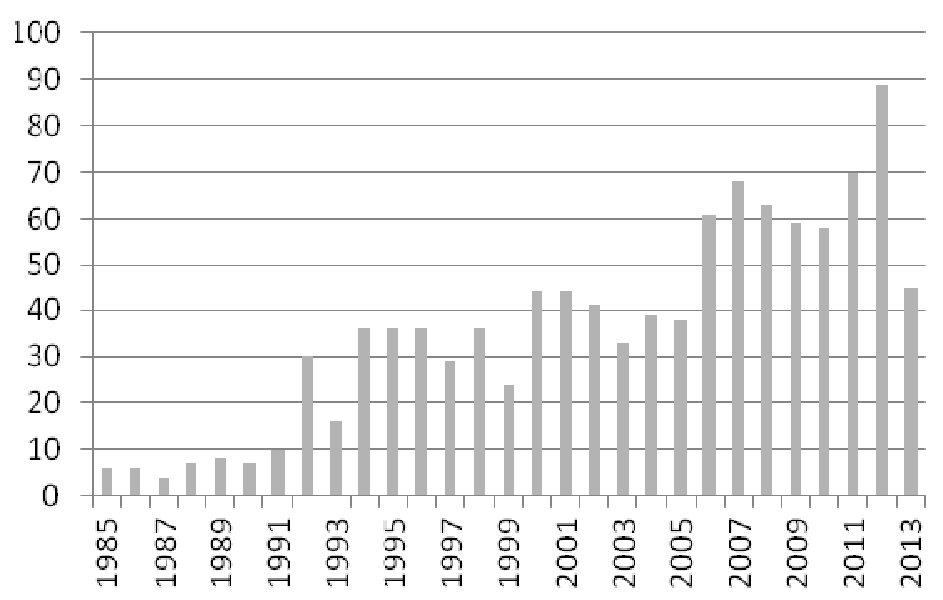

La figura 2 mostra questo tipo di informazione. In particolare, sull'asse di sinistra vengono misurati i contributi del dataset, mentre sull'asse di destra le pubblicazioni complessive nelle 89 riviste tenute in considerazione e presenti su ISI-WoS. Tali risultati ci permettono di concludere che, se in generale il numero delle pubblicazioni per anno è piuttosto stabile - fatta eccezione per l'impennata che si rileva negli ultimi anni a causa dell'apertura di ISI a nuovi volumi e riviste - nel caso del nostro dataset l'andamento è crescente.

Figura 2 - Numero pubblicazioni dataset ricerca e numero complessivo di pubblicazioni (per anno)

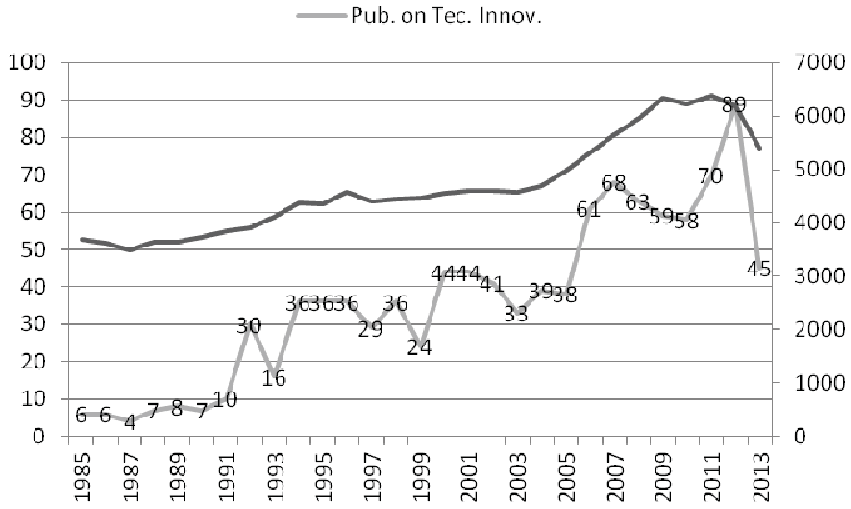


Il secondo indicatore descrittivo utilizzato nella nostra analisi è il numero di citazioni per anno. Come mostrato dalla figura 3, il numero di citazioni cresce in misura quasi esponenziale nel corso degli anni. Tale andamento può essere considerato come un indicatore dell'impatto di questi contributi sulla comunità scientifica nel suo complesso (in base alle informazioni presenti nei database di ISI-WoS).

Anche in questo caso i dati ottenuti per l'anno 2013 sono da considerarsi parziali. Tra i contributi del dataset più citati, 4 hanno ricevuto oltre 500 citazioni: Teece (1986) con 1894 citazioni, Shane (2000) con 711, Christensen e Bower (1996) con 511 e infine Benner e Tushman (2003) con 503 citazioni (vedi anche figura 4). Il contributo di Teece (1986) è stato pubblicato su 'Research Policy' - journal appartenente al TIM ranking -, gli altri tre contributi sono stati tutti pubblicati su riviste del FT45 ranking. Teece (1986), Shane (2000) e Christensen e Bower (1996), affrontano la tematica dell'innovazione tecnologica con una visione più strategica e imprenditoriale che organizzativa. Teece (1986), per esempio, spiega la possibilità di trarre profitti da un'innovazione, in base alla disponibilità di determinati asset complementari, quali competenze di marketing, supporto post-vendita, sistemi produttivi competitivi. Shane (2000) mostra come gli imprenditori siano fortemente influenzati dalle precedenti esperienze e dal percorso educativo/formativo nel riconoscere opportunità di business. La scoperta di un'opportunità di business, tramite la quale sfruttare tecnologie innovative, dipende dunque dalla distribuzione delle informazioni nella società. Christensen e Bower (1996) dimostrano come una delle ragioni principali per cui alcune imprese perdono le loro posizioni di leadership nel settore a fronte di certi tipi di cambiamento tecnologico ha poco a che fare con la tecnologia stessa, con il suo grado di novità o difficoltà, con le competenze o l'esperienza dell'impresa. Piuttosto, la causa del fallimento è rappresentata dall'aver ascoltato le richieste dei clienti e dall'aver conseguentemente limitato le strategie dell'impresa. Il contributo di Benner e Tushman (2003), invece, si rivolge a un pubblico interessato a fenomeni sia strategici che organizzativi. In particolare, gli autori estendono la letteratura sulla gestione dei processi sviluppando un modello contingente e le relative proposizioni su come le pratiche di gestione del processo - ad esempio il Total Quality Management (TQM) - influenzano sia l'innovazione tecnologica che l'adattamento organizzativo. Il contributo più citato tra quelli pubblicati sulle riviste di matrice sociologica è - con 60 citazioni - quello di Brown (1995) su 'Work Employment and Society'. L'autore considera l'innovazione tecnologica uno dei fattori determinanti la crescente doman- 
da di capitale culturale associata all'accesso della classe media alle carriere burocratiche che si registra negli anni ' 90 . Nell'ambito di questo processo, le forme fino ad allora dominanti di capitale culturale vengono svalutate a causa del cambiamento dei modelli di controllo simbolico all'interno delle organizzazioni.

Ulteriori informazioni rilevanti sono quelle relative alla distribuzione delle pubblicazioni per università di appartenenza degli autori (tabella 4) e per stato in cui è collocata l'università a cui gli autori sono affiliati (tabella $5)$.

In particolare, emerge una netta prevalenza di università nord americane e britanniche, ma anche un buon posizionamento dell'Italia che si colloca quinta tra gli stati con maggior numero di pubblicazioni sul tema dell'innovazione tecnologica e vanta una università tra le venti più produttive in materia: il Politecnico di Milano, infatti, si colloca al settimo posto a pari merito con la Stanford University, la Case Western Reserve University e la University of Illinois con un numero di pubblicazioni pari a 12.

Figura 3 - Numero di citazioni per anno

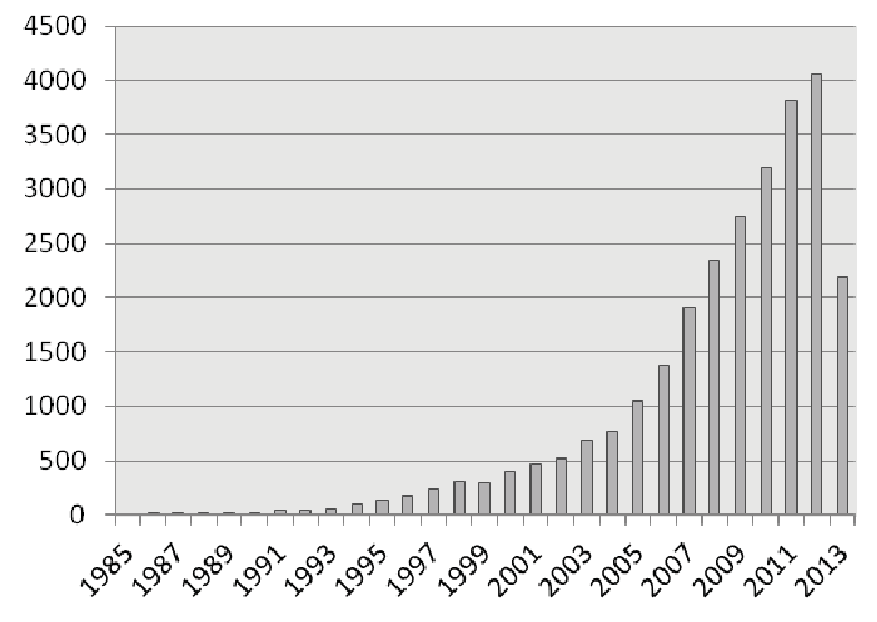


Tabella 4 - Distribuzione dei contributi per università di appartenenza degli autori (università con più di 10 pubblicazioni)

\begin{tabular}{lcc}
\hline \multicolumn{1}{c}{ UNIVERSITA } & $N^{\circ}$ PUBBLICAZIONI & $\%$ DI 1028 \\
\hline HARVARD UNIV & 26 & $2,5 \%$ \\
MIT & 22 & $2,1 \%$ \\
UNIV PENN & 17 & $1,7 \%$ \\
UNIV MINNESOTA & 16 & $1,5 \%$ \\
UNIV MANCHESTER & 15 & $1,5 \%$ \\
UNIV MARYLAND & 15 & $1,5 \%$ \\
UNIV UTRECHT & 15 & $1,5 \%$ \\
RENSSELAER POLYTECH INST & 14 & $1,4 \%$ \\
GEORGIA INST TECHNOL & 13 & $1,3 \%$ \\
MICHIGAN STATE UNIV & 13 & $1,3 \%$ \\
NYU & 13 & $1,3 \%$ \\
UNIV CALIF BERKELEY & 13 & $1,3 \%$ \\
CASE WESTERN RESERVE UNIV & 12 & $1,2 \%$ \\
POLITECN MILAN & 12 & $1,2 \%$ \\
STANFORD UNIV & 12 & $1,2 \%$ \\
UNIV ILLINOIS & 12 & $1,1 \%$ \\
UNIV CAMBRIDGE & 11 & $1,1 \%$ \\
UNIV SUSSEX & 11 & $1,1 \%$ \\
CHALMERS & 11 & $1,0 \%$ \\
UNIV LONDON IMPERIAL COLL SCI & 10 & $1,0 \%$ \\
TECHNOL MED & & \\
\hline
\end{tabular}

Tabella 5 - Distribuzione dei contributi per stato in cui è collocata l'università di appartenenza degli autori (stati con più di 10 pubblicazioni)

\begin{tabular}{|c|c|c|}
\hline STATO & $N^{\circ}$ PUBBLICAZIONI & $\% D I 1028$ \\
\hline USA & 410 & $40,1 \%$ \\
\hline ENGLAND & 130 & $12,1 \%$ \\
\hline CANADA & 64 & $6,2 \%$ \\
\hline NETHERLANDS & 60 & $5,9 \%$ \\
\hline ITALY & 56 & $5,6 \%$ \\
\hline SPAIN & 48 & $4,8 \%$ \\
\hline GERMANY & 43 & $4,3 \%$ \\
\hline PEOPLES R CHINA & 43 & $4,3 \%$ \\
\hline TAIWAN & 38 & $3,8 \%$ \\
\hline SOUTH KOREA & 26 & $2,5 \%$ \\
\hline SWEDEN & 26 & $2,4 \%$ \\
\hline SWITZERLAND & 24 & $2,4 \%$ \\
\hline FRANCE & 23 & $2,2 \%$ \\
\hline AUSTRALIA & 23 & $2,0 \%$ \\
\hline
\end{tabular}

(segue) 


\begin{tabular}{|c|c|c|}
\hline STATO & $N^{\circ}$ PUBBLICAZIONI & $\%$ DI 1028 \\
\hline JAPAN & 20 & $2,0 \%$ \\
\hline AUSTRIA & 16 & $1,6 \%$ \\
\hline BRAZIL & 14 & $1,4 \%$ \\
\hline FINLAND & 14 & $1,4 \%$ \\
\hline SINGAPORE & 14 & $1,4 \%$ \\
\hline INDIA & 13 & $1,3 \%$ \\
\hline BELGIUM & 11 & $1,1 \%$ \\
\hline DENMARK & 11 & $1,1 \%$ \\
\hline
\end{tabular}

\subsection{Analisi dei contenuti}

Le 1028 pubblicazioni sono state analizzate anche attraverso gli strumenti della Social Network Analysis.

La prima analisi eseguita focalizza l'attenzione sugli studiosi e sui ricercatori che hanno prodotto i 1028 contributi inclusi nel dataset mirando a identificare le relazioni tra gli autori più citati (co-authorship), la loro produttività, ovvero per ciascuno il numero di lavori presenti nel dataset, e il loro impatto sulla comunità scientifica rappresentato dal numero complessivo di citazioni ricevute per tali contributi. Di conseguenza, nella figura 4 sono riportate due tipologie di nodi: i) i lavori più citati presenti nel dataset (nodo dalla forma quadrata) e gli autori che hanno ricevuto il maggior numero di citazioni (nodo dalla forma circolare), dato dalla somma delle citazioni delle relative pubblicazioni presenti nel dataset. Impostando come soglia 200 citazioni, sono stati selezionati 18 paper su 1028 e i relativi 30 autori sui circa 1700 complessivi. Per ogni nodo, all'inizio della relativa etichetta, è riportato tra parentesi quadre ("[]") il numero di citazioni; mentre per ogni autore, dopo il nome, è indicato il numero di contributi pubblicati e appartenenti al dataset preceduto dal simbolo "\#”. Dalla figura 4 si evince come Teece sia il più citato tra gli autori e ciò è evidenziato dal totale delle citazioni complessivamente ricevute (2037) dai suoi 4 paper presenti nel dataset, anche se su un solo contributo (il più citato nel set) ne riceve la maggior parte (1894). In generale, nel dataset considerato, tutti gli autori hanno un solo contributo che ha ricevuto un numero considerevole di citazioni (oltre 200), a eccezione di Benner che ne ha due. Sempre dalla figura 4 è possibile evidenziare la presenza o meno di collaborazioni (coauthorship) tra autori relativamente ai lavori che hanno avuto un maggiore impatto sulla comunità scientifica. Infine va menzionato che non sempre gli autori più produttivi sono quelli più citati: infatti nel grafo rappresentato in 
figura 4 sono presenti autori con al massimo 4 lavori mentre altri autori, pur avendo un numero maggiore di pubblicazioni, ne sono stati esclusi per il basso numero di citazioni ricevute. A titolo esemplificativo è possibile considerare il caso di Linton che, pur essendo risultato il più produttivo (ben 9 contributi all'interno del dataset), non è raffigurato nel grafico avendo ricevuto, complessivamente, soltanto 87 citazioni.

Figura 4 - Network degli autori/paper più citati

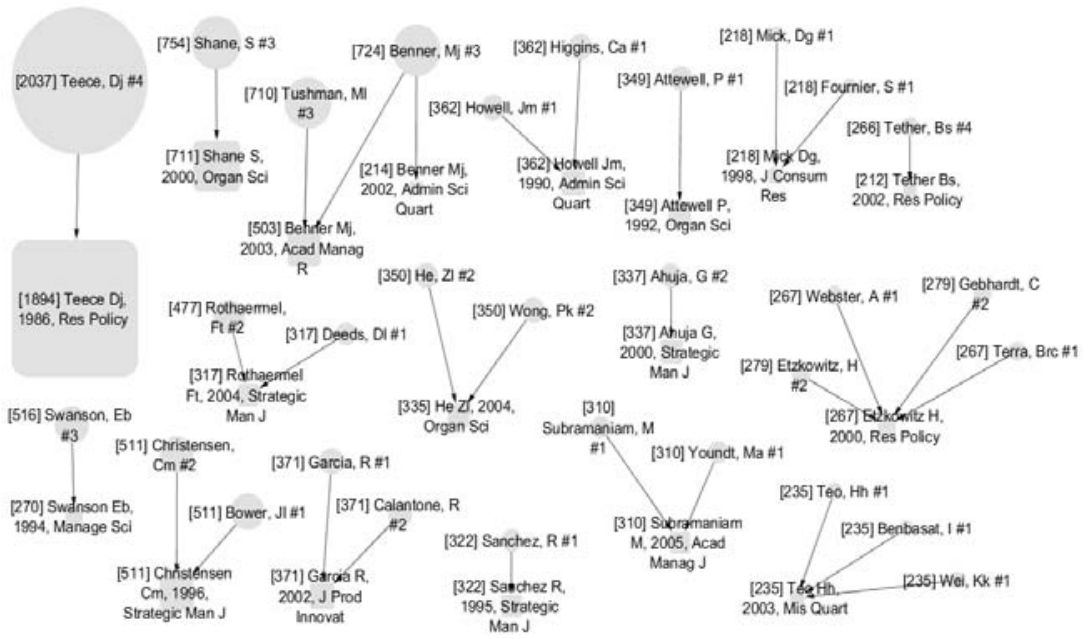

Dopo aver analizzato sia la produttività degli autori, sia l'impatto delle loro pubblicazioni sulla comunità scientifica, è stata esaminata la distribuzione dei contributi in termini di categorie (Web of Science subject categories - figura 5). Queste categorie vengono assegnate dallo staff di ISI sulla base delle caratteristiche della rivista di pubblicazione come, ad esempio, il titolo del journal e il tipo e l'andamento delle citazioni

Complessivamente, il campione esaminato appare equilibrato in termini di rappresentatività delle varie categorie: il dataset delle 1028 pubblicazioni può essere suddiviso in $18 \mathrm{Web}$ of Science subject categories. Le tre categorie più diffuse sono 'management' ( 865 contributi; $84,1 \%$ del totale), 'business' (431; 41.9\%) e 'engineering industrial' (293; 28.5\%), seguite da 'planning development' (250;24.3\%), 'operations research \& management sciences' $(245 ; 23.8 \%)$. La dimensione dei nodi rappresentati in figura 5 riflette questa informazione. Il posizionamento delle categorie 'management' e 'business' in cima alla lista, implica una tradizione e un focus più manageriale che tecnico o sociologico all'interno della letteratura 
sull'innovazione tecnologica. Questo risultato supporta, inoltre, la decisione di combinare diversi ranking di riviste. In aggiunta, la figura mostra $\mathrm{i}$ legami tra le varie categorie e, nello specifico, lo spessore degli archi indica quante pubblicazioni sono classificate in entrambe le categorie. Possiamo dunque osservare che 308 contributi sono assegnati sia alla categoria 'management' che alla categoria 'business'; altre due coppie di categorie, inoltre, sono collegate da più di 200 pubblicazioni - 'management' e 'engineering industrial' (293), 'management' e 'operations research \& management sciences' (245). La distribuzione delle pubblicazioni tra una varietà di categorie - includendo anche 'Multidisciplinary Sciences','Ethics', 'Business Finance', 'Applied Psychology', 'Sociology', 'Social Work', 'Industrial Relations Labor', 'Social Sciences Interdiscipli$n a r y$ ' - ci da un'idea della multidisciplinarietà dell'area di ricerca legata all'innovazione tecnologica. Questa diversità all'interno delle subject categories dovrebbe suggerire agli studiosi interessati alla comprensione e all'approfondimento del fenomeno dell'innovazione tecnologica di allargare la prospettiva di indagine e di includere nelle loro ricerche anche riviste con un focus più ampio e, tendenzialmente, considerando anche prospettive diverse.

Figura 5 - Network delle subject categories

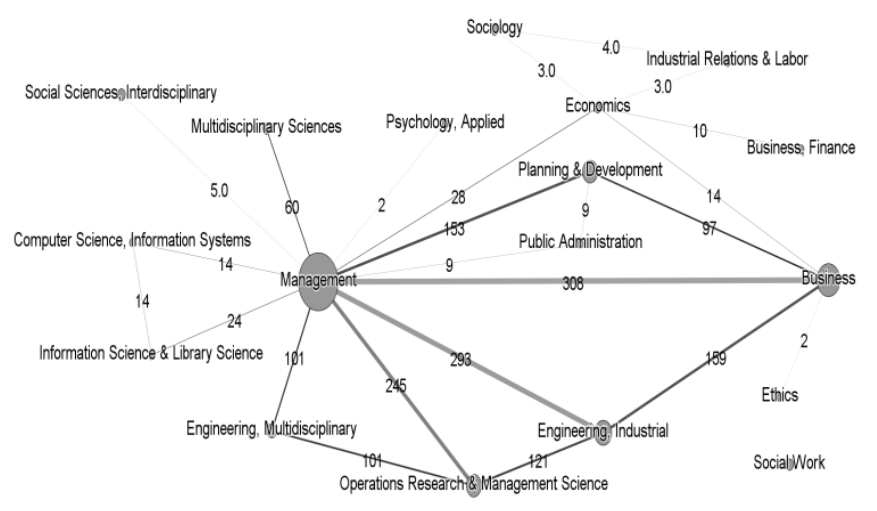

Al fine di approfondire quanto emerso dall'analisi delle subject categories, sono state esaminate anche le aree di ricerca che ISI-WoS attribuisce a ogni pubblicazione in base al contributo e al campo specifico dello studio. Le evidenze ottenute convalidano quanto già emerso: l'area 'business \& economics' (1017 pubblicazioni; 98,9\% del totale) si conferma, infatti, essere l'area più diffusa e con un limitato numero di contributi in comune con 
le aree 'sociology' (solo 4), 'psychology' e 'social science-other topics' (solo 2).

Sulla base di quanto emerge dalla figura 5 si conferma la multidisciplinarietà della ricerca sull'innovazione tecnologica ma viene anche scongiurato il rischio di considerare la sola prospettiva dell'Information Techno$\log y$ (IT).

Tuttavia, poiché le subject categories sono definite da ISI-WoS, si è deciso di condurre una ulteriore analisi basata sulle parole chiave definite dagli autori per ogni contributo. Le keywords, infatti, riflettono in maniera più accurata il focus di ogni singolo contributo fornendo una panoramica più dettagliata sulle tematiche della ricerca sull'innovazione tecnologica. Analizzando il trend delle occorrenze delle varie keywords associate ai paper del dataset (Figura 6) emerge che prima delle 30 occorrenze (indicate dal punto rosso nella figura) l'andamento tende a essere lineare mentre, subito dopo, si evidenzia una crescita più rapida e più consistente.

Figura 6-Il trend delle occorrenze delle parole chiave

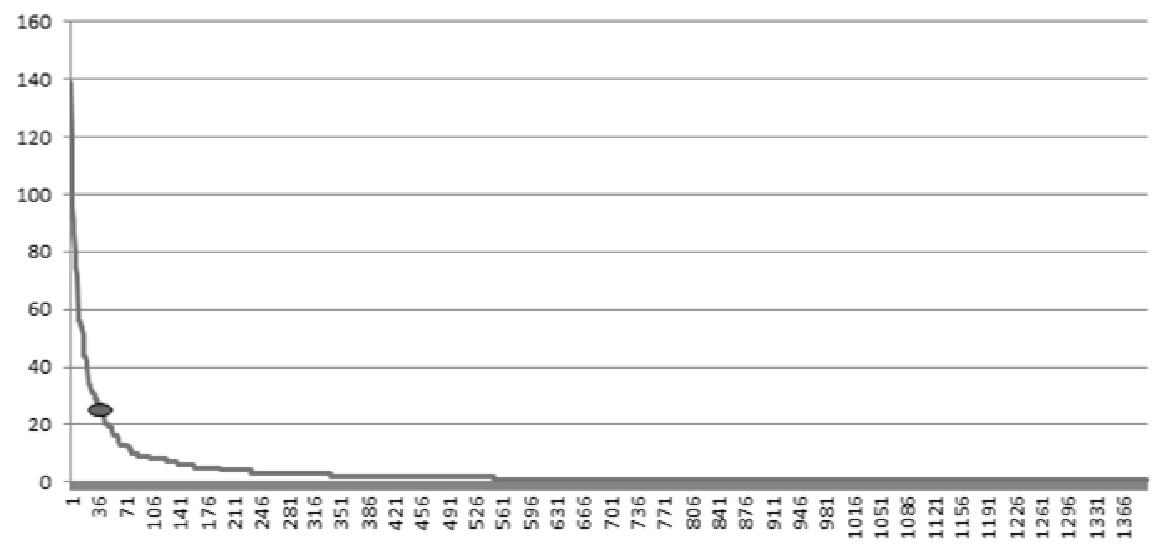

È stato così identificato l'insieme delle 32 keywords più utilizzate nel dataset, ovvero quelle che presentano occorrenze uguali o superiori a $30 \mathrm{e}$ che rappresentano, inoltre, il 36\% delle occorrenze totali. Le 32 parole chiave selezionate e i nodi del network sono illustrati in figura 7. La dimensione dei nodi (e delle rispettive etichette) rappresenta la ricorrenza delle parole chiave all'interno del dataset. Ciò che emerge è che 'performance' (139), 'technological innovation' (117), 'research and development' (119) sono le tre keyword più utilizzate. Tra le parole chiave c'è anche 'technological-innovation' (90 ripetizioni) che può essere vista come sinonimo di 
'technological innovation'. Considerando la ricorrenza di entrambe, si raggiunge il valore più alto (207). Il legame tra due parole chiave indica che esse sono menzionate nella stessa pubblicazione, mentre lo spessore è relativo al numero di contributi nei quali la coppia appare. La coppia di ke$y$ word più popolare è 'performance/technological innovation' che si ripete 37 volte; entrambe le coppie 'performance/research and development' e 'technological-innovation/research and development' ricorrono 32 volte. La parola chiave 'performance' è spesso abbinata a 'firms' (26 volte); ricorre 23 volte anche con le keyword 'management', 'industry' e 'knowledge'. Tematiche legate a 'technological innovation', invece, sono: 'product development' (la coppia si ripete 20 volte), 'firms' ( 22 volte) e 'knowledge' (21 volte). Infine, la parola chiave 'research and development' è menzionata 21 volte insieme a 'absorptive capacity'.

Figura 7-Network dell'occorrenza delle parole chiave

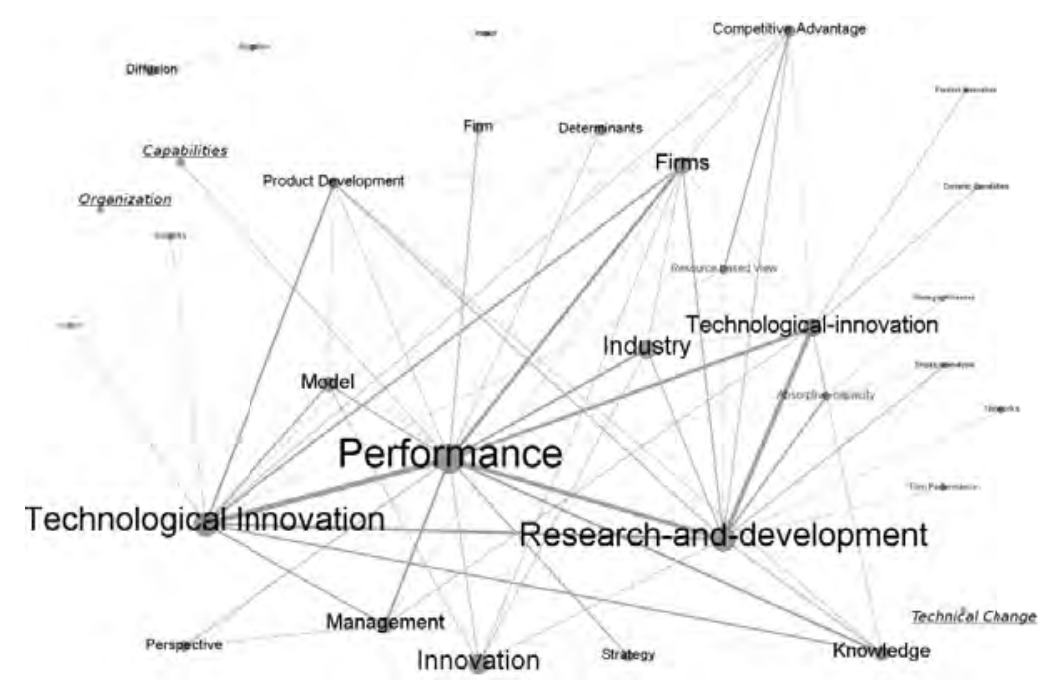

In generale, i risultati ottenuti con riferimento all'analisi della occorrenza delle keyword mostrano come alcune questioni e aspetti legati alla ricerca sull'innovazione tecnologica sono più affrontati di altri. In particolare, in base alla nostra analisi, vale la pena evidenziare che il legame tra innovazione tecnologica e parole chiave quali 'organization', 'capabilities' e 'technical change', ha, fino a ora, ricevuto scarsa attenzione nella letteratura internazionale, anche in quella più tipicamente manageriale (per esem- 
pio, nei 4 contributi più citati del dataset, solamente in quello di Benner e Tushman (2003), è presente la parola chiave "organizations").

La scarsa attenzione dedicata a esplorare aspetti e relazioni legate maggiormente all'organizzazione e al lavoro viene confermata nel tempo: andando ad analizzare le keyword più ricorrenti in ciascuna delle fasi considerate in questo studio (emergente, interesse crescente e rafforzamento), infatti, non si ottengono differenze significative. É inoltre sorprendente l'assenza di tematiche tradizionalmente legate all'innovazione tecnologica nell'ambito degli studi organizzativi, ovvero 'design', 'employment', 'task' o, più in generale, 'job' (Bell, 1967; Butera e Thurman, 1984; Hage e Aiken, 1969). Anche focalizzando l'attenzione sui soli journals di matrice sociologica vengono confermate queste risultanze: tra le parole più citate troviamo, infatti, 'technological-innovation' e 'politics' seguite poi da 'innovation', 'technology', 'context', 'strategy' e 'gender'.

\subsection{La ricerca sull'innovazione tecnologica in Italia}

Le analisi condotte sulle 1028 pubblicazioni sono state replicate sui soli contributi italiani al fine di comprendere l'andamento e i contenuti della ricerca sull'innovazione tecnologica con particolare riferimento al nostro paese. In questa ricerca si è considerata come pubblicazione 'italiana' quella avente almeno uno degli autori affiliato presso un'università, un istituto di ricerca o un qualsiasi altro ente italiano. Data questa definizione sono stati identificati 56 contributi scientifici italiani, ovvero circa il 5,4\% del dataset complessivo.

Nell'ambito dell'analisi descrittiva sono stati esaminati diversi indicatori tra cui l'evoluzione del numero delle pubblicazioni e delle citazioni per anno (vedi figura 8). Con riferimento alle prime e rispetto a quanto visto a livello complessivo, a livello nazionale la fase emergente - il primo periodo di ricerca identificato a livello internazionale (1985-1991) - sembrerebbe essere non individuabile o, comunque, posticipato. Il primo contributo italiano risale, infatti, al 1988 e non si riscontrano altri contributi sino al 1992. Gli ultimi anni, invece, si confermano essere i più proliferi da un punto di vista scientifico: il numero massimo di pubblicazioni - pari a 6 - si ha nel 2008, anno a partire dal quale non si scende mai al di sotto dei 3 contributi annui. Anche nel 2013, anno per cui i risultati sono da considerarsi parziali, sono state identificate 2 pubblicazioni lasciando presumere la possibilità di una loro crescita nei mesi successivi ad agosto, mese in cui è stata condotta 
la rilevazione. Le tre riviste in cui è stato pubblicato il maggior numero di contributi sono Research Policy con 11 pubblicazioni (19.6\% dei contributi italiani) seguita da Technological Forecasting and Social Change e Technovation, rispettivamente con $9(16.1 \%)$ e $8(14.3 \%)$ pubblicazioni scientifiche. Se sull'asse di sinistra della figura 8 è indicato il numero delle pubblicazioni, su quello di destra troviamo la misura del secondo indicatore descrittivo considerato, ovvero il numero delle citazioni per anno. Il trend italiano, pur se con qualche anno di ritardo rispetto all'inizio del periodo di analisi considerato, si conferma essere in crescita nel corso degli anni, in special modo nell'ultimo triennio. Tra le 1028 pubblicazioni troviamo che $\mathrm{i}$ contributi italiani più citati sono quello di Chiesa, Coughlan e Voss pubblicato su Journal of Product Innovation Management nel 1996 (139 citazioni), quello di Bonaccorsi e Piccaluga (1994) nella rivista $R \& D$ Management (90 citazioni) e, da ultimo, l'articolo di Sirilli e Evangelista pubblicato nel 1998 su Research Policy (85 citazioni).

Figura 8-Numero di pubblicazioni/citazioni contributi italiani

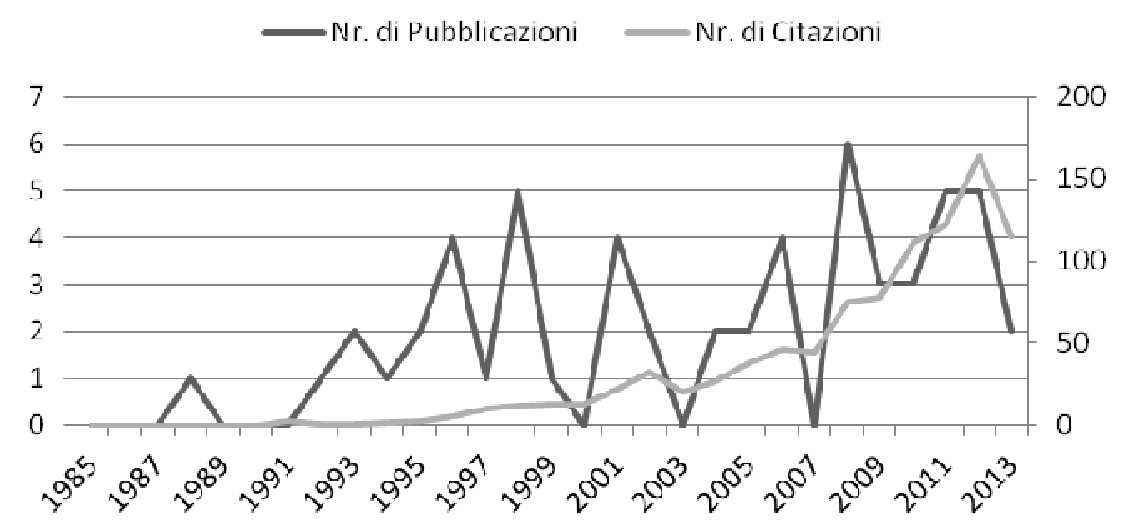

Sempre nell'ambito dell'analisi descrittiva, è stata esaminata la distribuzione geografica delle collaborazioni tra autori. In particolare, dalla tabella 6 emerge che tra le 56 pubblicazioni italiane, ben 35 sono state prodotte esclusivamente da autori italiani. Le collaborazioni estere più significative dei ricercatori italiani si hanno, invece, con autori appartenenti a università o enti di ricerca statunitensi (13\% delle pubblicazioni) e con autori che svolgono la loro attività di ricerca in Inghilterra ( $9 \%$ dei contributi).

Tra gli autori italiani più produttivi troviamo Chiesa con 6 pubblicazioni (il 10,7\% dei contributi italiani), Coccia ed Evangelista entrambi con 5 
pubblicazioni $(8,9 \%)$ e, da ultimo, Esposito con 4 (7,1\%). La figura 9, ottenuta ricorrendo agli strumenti della SNA, mostra le co-authorship relative esclusivamente ai contributi italiani. Dalla figura è possibile osservare che sono tre le coppie di autori che hanno collaborato su più di una pubblicazione. Inoltre, i due autori che si trovano al centro di network collaborativi Chiesa e Evangelista - sono due degli autori citati come particolarmente produttivi.

Attraverso gli strumenti della SNA, anche per i contributi scientifici italiani, è stata esaminata la distribuzione in termini di categorie (Web of Science subject categories - figura 10).

Tabella 6 - Distribuzione geografica delle collaborazioni tra autori italiani e stranieri per stato in cui è collocata l'università di appartenenza degli autori

\begin{tabular}{lcc}
\hline \multicolumn{1}{c}{ STATO } & $N^{\circ}$ PUBBLICAZIONI & $\%$ DI 56 \\
\hline ITALY & 35 & $63 \%$ \\
USA & 7 & $13 \%$ \\
ENGLAND & 5 & $9 \%$ \\
AUSTRIA & 2 & $4 \%$ \\
GERMANY & 2 & $4 \%$ \\
SWEDEN & 2 & $4 \%$ \\
FINLAND & 1 & $2 \%$ \\
IRELAND & 1 & $2 \%$ \\
TAIWAN & 1 & $2 \%$ \\
\hline
\end{tabular}

Complessivamente, le 56 pubblicazioni possono essere suddivise in 9 Web of Science subject categories, rispetto alle 18 in cui, invece, erano state suddivise le 1028 pubblicazioni dell'intero dataset. Le due categorie più diffuse si confermano essere 'management' (47 pubblicazioni; $87.9 \%$ dei contributi italiani)'business' $(22 ; 39.3 \%)$. Queste due categorie sono poi seguite da 'planning development' (20;35.7\%), 'engineering industrial' $(19 ; 33.9 \%)$ e, da ultimo, 'operations research \& management sciences' $(15 ; 26.8 \%)$.

Il solo avvicendamento di posizione si ha, quindi, tra le categorie 'planning development' e 'engineering industrial' che nel caso italiano diventano, nell'ordine, la terza e la quarta categoria. Inoltre, considerando lo spessore degli archi di collegamento tra le diverse categorie, è possibile osservare che 19 contributi sono assegnati sia alla categoria 'management' che alla categoria 'engineering industrial'; altre due coppie di categorie sono collegate da più di 10 contributi - 'management' e 'operations research \& management sciences' (15), 'management' e 'business' (13). 
Così come fatto per il dataset complessivo, anche per i contributi italiani si è proceduto a verificare la coerenza con le risultanze derivanti da un approfondimento fatto sulle aree di ricerca. In linea sia con quanto illustrato per il dataset complessivo, sia con quanto visto per mezzo dell'analisi delle subject categories, i campi di studio e ricerca più diffusi risultano essere 'business \& economics' attribuito alla totalità dei 56 contributi italiani qui considerati, seguito da 'engineering' ( 25 pubblicazioni; $44,6 \%$ del totale delle pubblicazioni italiane) e 'public administration' (20; 35,7\%).

Figura 9 - Collaborazioni tra autori nei contributi italiani

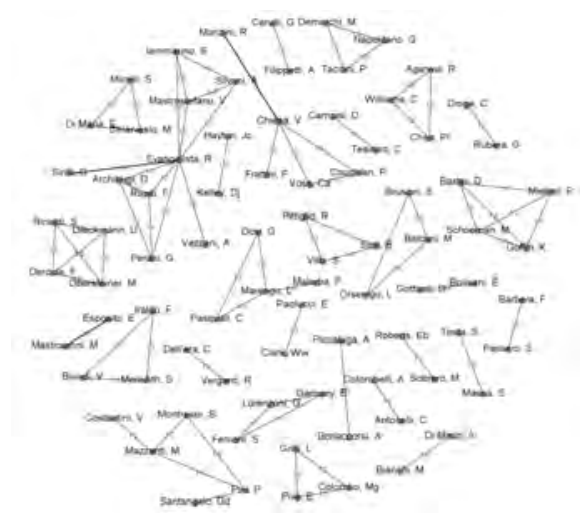

Figura 10 - Network delle subject categories nei contributi italiani

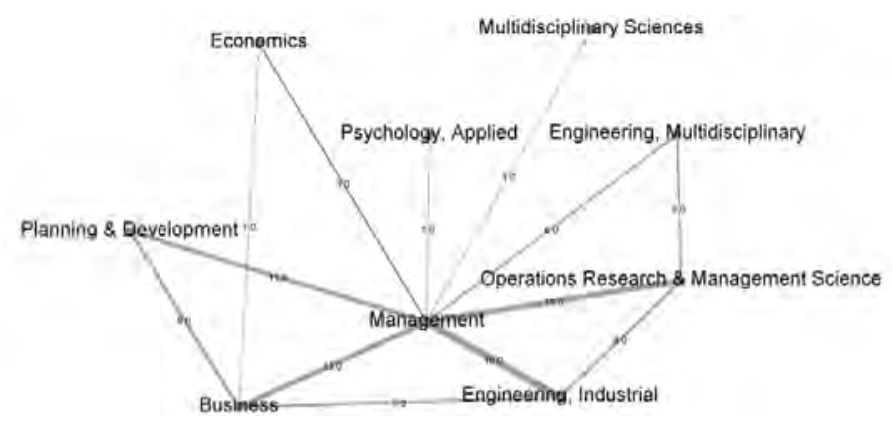

Un'ulteriore analisi, come per l'intero dataset, ha avuto l'obiettivo di presentare la varietà, la diversità e la ricorrenza delle keyword nei contributi italiani, nonché di individuare eventuali relazioni tra le stesse. Riflettendo i contenuti principali di ciascun contributo ed essendo indicate direttamente 
dagli autori, le parole chiave favoriscono l'individuazione delle tematiche su cui la ricerca sull'innovazione tecnologica si è maggiormente concentrata in questi ultimi decenni. Le 141 keyword presenti nei 56 contributi sono mostrate nel grafico che segue (figura 11). Dall'analisi emerge che 'technological innovation' (10 ripetizioni) si conferma essere una delle keyword più utilizzate, insieme a 'innovation' (sempre 10 ripetizioni) mentre la terza parola chiave più ricorrente è 'technology'. Seguono - tutte con due ripetizioni - le seguenti keyword: 'economic impact', 'information tecnhology', 'patterns of technological innovation', 'R\&D', 'Smes', 'Teece', 'technometrics'. Relativamente alla ricorrenza delle coppie, invece, solamente una di quelle mostrate nel network si ripete per due volte e si tratta della coppia 'innovation/Teece'; tutte le altre coppie hanno ricorrenza pari a 1. Questo sottolinea la pluralità delle tematiche - connesse al focus principale della presente ricerca - affrontate dai 56 contributi. Dalla figura 11 emerge, inoltre, che le tematiche maggiormente legate a 'technological innovation' appaiono essere 'innovation management in multinationals', 'international $R \& D$ structures', 'global $R \& D$ ', 'internationalisation of technology', 'global management of technology'. Un'analisi più approfondita del network delle parole chiave sotto raffigurato mostra la rilevanza di altri temi legati alle keywords 'innovation' e 'technology' quali: 'economic impact', 'sustainability', 'environmental policies', 'transaction costs', 'export perfomance', 'European Union'. Dai risultati ottenuti con riferimento all'analisi delle keyword nelle 56 pubblicazioni italiane analizzate sembra emergere che il tema dell'innovazione tecnologica sia maggiormente correlato a tematiche macro economiche-sociali evidenziando, ancora una volta, la scarsa attenzione prestata al legame tra innovazione tecnologica, organizzazione e lavoro.

Figura 11 - Network della ricorrenza delle parole chiave nei contributi italiani

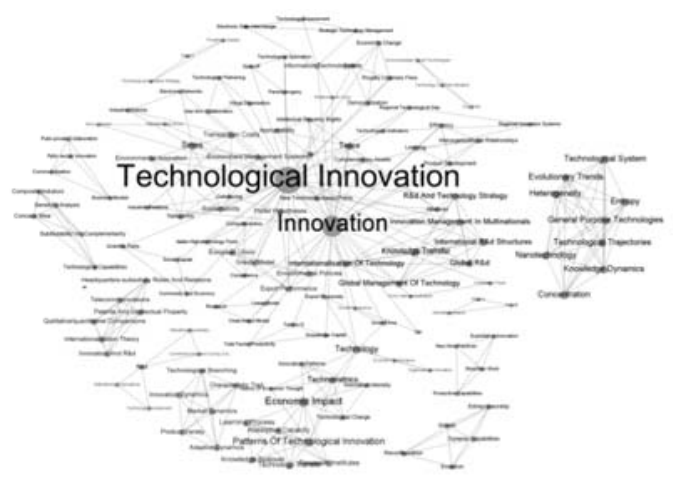

66

Copyright $\odot$ FrancoAngeli

N.B: Copia ad uso personale. Ė vietata la riproduzione (totale o parziale) dell'opera con qualsiasi mezzo effettuata e la sua messa a disposizione di terzi, sia in forma gratuita sia a pagamento. 


\section{Discussioni e conclusioni}

Questo studio offre un'ampia panoramica sulla letteratura relativa al tema dell'innovazione tecnologica con un approfondimento dedicato al contesto italiano.

L'analisi condotta e qui presentata è stata motivata da due osservazioni: in primo luogo, l'innovazione tecnologica e le sue determinati sono rimaste - nel corso degli ultimi anni - una tra le tematiche maggiormente dibattute nella letteratura accademica; in seconda istanza, questa stessa letteratura sembra aver trascurato il ruolo e l'impatto dell'innovazione tecnologica sull'organizzazione e sul lavoro. È proprio partendo da queste considerazioni che l'analisi è stata impostata con un duplice obiettivo: fornire una panoramica della letteratura esistente sull'innovazione tecnologica e identificare aree di studio e di ricerca esistenti o potenziali volte ad approfondire il legame tra innovazione tecnologica, organizzazione e lavoro.

Sebbene studi precedenti abbiano già esplorato lo stato dell'arte della letteratura sull'innovazione tecnologica, la presente analisi estende e integra questi risultati sia utilizzando un set di riviste più ampio e metodi bibliometrici avanzati di analisi (SNA), sia fornendo un approfondimento relativo al contesto italiano. Il nostro paese appare, infatti, come una realtà particolarmente produttiva collocandosi al quinto posto tra gli stati con maggior numero di pubblicazioni sul tema dell'innovazione tecnologica.

Con riferimento al dataset complessivo - 1028 pubblicazioni scientifiche selezionate da 89 riviste accademiche internazionali -, l'analisi descrittiva e la social network analysis condotte mostrano una crescita significativa della letteratura sull'innovazione tecnologica nel corso degli anni, la multidisciplinarietà del settore di ricerca, così come la rilevanza dei contributi di 'management' e 'business \& economics' rispetto a tematiche strettamente tecniche. In particolare, l'analisi della ricorrenza delle parole chiave sottolinea la necessità di una maggiore esplorazione, a livello sia teorico che empirico, dell'innovazione tecnologica nell'ambito della vita organizzativa. In altre parole, a una forte enfasi sul bisogno e sulla nascita dell'innovazione sembrerebbe corrispondere una minore attenzione prestata a fenomeni quali il funzionamento e l'impatto della stessa sulle competenze, sul comportamento degli attori organizzativi e, più in generale, sul lavoro. Con riferimento a quest'ultimo, infatti, anche le riviste internazionali di matrice sociologica considerate in questo studio non hanno evidenziato un'attenzione particolare al tema, trascurando legami sicuramente importanti quali, per esempio, quelli tra innovazione tecnologica e cultura orga- 
nizzativa, lavoro a distanza, flessibilità del lavoro e gestione delle risorse umane.

Il focus sui contributi italiani conferma il trend complessivo relativo al numero di pubblicazioni e citazioni, seppur con un lieve sfasamento temporale. I dati raccolti con riferimento al contesto italiano mostrano, inoltre, la tendenza degli autori italiani a collaborare con connazionali o, nel caso di collaborazioni con autori di università straniere, a privilegiare il contesto nord-americano e anglosassone. Non ci sono differenze significative rispetto al totale dei contributi analizzati per quanto riguarda la distribuzione per categorie e aree di ricerca, anzi viene confermata la marginalità della prospettiva dell'IT nell'ambito della letteratura sull'innovazione tecnologica. Infine, l'analisi delle keyword attribuite dagli autori alle 56 pubblicazioni italiane rafforza le conclusioni elaborate relativamente all'analisi del dataset complessivo, ovvero la scarsa attenzione dedicata al ruolo e all'impatto dell'innovazione tecnologica nell'ambito organizzativo, rimarcando in questo caso specifico addirittura una forte attenzione verso tematiche sociali ed economiche.

\subsection{Sviluppi futuri}

Partendo dall'insieme limitato dei contributi scientifici che, sino a oggi, hanno investigato il rapporto tra innovazione tecnologica e organizzazione, è possibile individuare alcuni futuri sviluppi di ricerca, sia teorica sia empirica, che potrebbero riguardare aspetti, pratiche e fenomeni a livello macro, meso e micro organizzativo.

A livello macro ricerche future potrebbero esplorare in che misura e come l'introduzione di un'innovazione tecnologica implica un adattamento e un conseguente cambiamento organizzativo. Non è difficile immaginare, infatti, come l'introduzione di innovazioni tecnologiche influenzi gli assetti organizzativi, le relazioni spaziali e temporali tra gli elementi fisici di un'organizzazione e la definizione dei suoi confini. Potrebbe essere interessante, pertanto, approfondire il legame tra innovazione tecnologica e la diffusione di strutture organizzative più flessibili (Harris, 2006) o la formazione di relazioni di collaborazione tra imprese (per esempio la formazione di reti di imprese - Staropoli, 1998; Sen e Egelhoff, 2000). Un' altra area di ricerca potenzialmente interessante e ancora poco esplorata è rappresentata dal legame tra lo sviluppo e l'introduzione di un'innovazione tecnologica e 
il fenomeno dell'apprendimento organizzativo (Van de Ven e Polley, 1992).

A livello meso, sarebbe interessante approfondire la relazione (bidirezionale) tra dinamiche di gruppo e processo di selezione/adozione di una innovazione tecnologica e comprendere, quindi, sia come le caratteristiche a livello meso possano favorire l'innovazione, sia come quest'ultima influenzi a sua volta le relazioni tra attori organizzativi. Relativamente al primo verso della relazione, nel dataset analizzato si trovano alcuni esempi. Vi sono, infatti, alcuni studi relativi alle caratteristiche di livello meso favorevoli al processo di innovazione, che possono riguardare sia aspetti informali, come la coesione dei gruppi di lavoro (Lloréns Montes et al., 2005), sia formali, come il ruolo dei team interfunzionali (Santa et al., 2011). Sempre in quest'ambito, ci sono alcuni lavori relativi alla gestione della conoscenza e alla sua condivisione (formale e informale) nell'ambito dei team e a come questa possa influenzare sia il processo decisionale riguardo l'adozione di un'innovazione (Wu et al., 2010), sia il suo successo a lungo termine (Bong et al., 2004). Prendendo spunto dagli studi appena citati sarebbe interessante approfondire l'altro verso della relazione, a oggi ancora poco esplorato: cioè come l'innovazione tecnologica possa avere un impatto sulle dinamiche di gruppo e sulla gestione della conoscenza, nel breve e nel lungo periodo, arrivando eventualmente a identificare anche alcuni aspetti di reciproca influenza.

Da ultimo, spazi di approfondimento possono essere trovati anche a livello micro-organizzativo. Ogni innovazione tecnologica o organizzativa, infatti, ridisegna l'ambiente organizzativo (Wingreen e Blanton, 2007) richiedendo un ri-adattamento tra persona e organizzazione (personorganization fit, Kristof, 1996). L'innovazione è da intendersi, in fondo, come un processo dinamico che produce impatti sia sullo sviluppo, sia sul mantenimento di determinate competenze e dei comportamenti organizzativi a esse associate. Di conseguenza, l'adattamento tra persona e organizzazione non può più essere valutato - in maniera statica - in un singolo momento nel tempo ma deve essere considerato come un processo dinamico che si ridefinisce continuamente. In particolare, a livello micro, l'innovazione tecnologica impatta sulla definizione di questo equilibrio richiedendo così la comprensione dei suoi effetti sullo sviluppo di nuove professionalità e sulle nuove competenze richieste (Katzy e Crowston, 2007), sulle capacità e sui tratti della personalità che più di altri possono assicurare il successo dell'innovazione (Williamson, Lounsbury e Han, 2013). 
Da un punto di vista metodologico, le aree di ricerca individuate potrebbero comportare l'utilizzo di altri strumenti di SNA sia per identificare cluster di lavori scientifici sulla base dei riferimenti bibliografici comuni (co-reference analysis - Börner, Penumarthy, Meiss, e Ke, 2006; White, Wellman e Nazer, 2004), sia per individuare le teorie basilari (theoretical building blocks) utilizzando le citazioni ricorrenti (co-citation analysis Small, 1973) anche conducendo analisi longitudinali sulle tre fasi identificate - emergente, interesse crescente, rafforzamento. L'analisi bibliometrica potrà essere utilizzata, inoltre, per esaminare l'evoluzione nel tempo del processo di creazione della conoscenza all'interno del set di contributi selezionati (cross-citation analysis - Nicolaisen, 2007; Za e Spagnoletti, 2013). Anche per mezzo di una text-analysis operata sul contenuto degli abstract, $\mathrm{i}$ lavori futuri potranno identificare tematiche nuove ed emergenti relativamente al rapporto tra innovazione tecnologica e organizzazione (a livello macro, meso e micro organizzativo). Infine, potrà essere condotta un'analisi qualitativa-interpretativa di un sotto insieme ristretto di contributi, identificati nella fase di analisi bibliometrica in quanto teorie basilari, oppure rappresentanti dei cluster. Le analisi condotte con riferimento alle sole pubblicazioni italiane potrebbero, inoltre, essere replicate considerando altre nazionalità al fine di evidenziare le peculiarità di altri paesi nell'ambito della ricerca sull'innovazione tecnologica.

Tra i limiti di questa ricerca vogliamo ricordare che, al fine di fornire un quadro d'insieme sulla letteratura esistente relativamente alla tematica dell'innovazione tecnologica, sono state identificate 89 riviste internazionali e, quindi, l'approfondimento sui contributi italiani è stato limitato ai lavori di autori italiani pubblicati su journal internazionali. Future ricerche potrebbero verificare la corrispondenza o meno delle tematiche trattate da autori italiani nelle pubblicazioni su riviste locali, piuttosto che su lavori monografici.

Complessivamente, questo articolo offre un'ampia analisi della letteratura sull'innovazione tecnologica e un approfondimento sulla realtà italiana palesando la necessità di studi futuri, con possibili implicazioni sia a livello teorico che pratico, volti a investigare la relazione tra innovazione tecnologica, organizzazione e lavoro. 


\section{Bibliografia di riferimento}

Ahuja, G., Lampert, C. M., Tandon, V. (2008), "Moving Beyond Schumpeter: Management Research on the Determinants of Technological Innovation", Academy of Management Annals, 2: 1-98.

Becheikh, N., Landry, R., Amara, N. (2006), "Lessons from Innovation Empirical Studies in the Manufacturing Sector: A Systematic Review of the Literature from 1993-2003", Technovation, 26: 644-664.

Bell, G.D. (1967), "Determinants of Span of Control”, American Journal of Sociology, 73: 100-109.

Benner, M. J., Tushman, M. L. (2003), "Exploitation, exploration, and process management: the productivity dilemma revisited", Academy of Management Review, 28: 238-256.

Bonaccorsi, A., Piccaluga, A. (1994), "A theoretical framework for the evaluation of university-industry relationships", $R \& D$ Management, 24: 229-247.

Bong, S. H., Lee, J., Gil, Y. (2004), "Effective team processes for technology internalisation with special emphasis on knowledge management: successful late starter, Samsung case", International Journal of Technology Management, 27: 16-39.

Börner, K., Penumarthy, S., Meiss, M., Ke, W. (2006), "Mapping the Diffusion of Scholarly Knowledge among Major U.S. Research Institutions", Scientometrics, 68: 415-426.

Brown, P. (1995), Cultural capital and social exclusion: some observations on recent trends in education, employment and the labour market. Work, Employment \& Society, 9: 29-51.

Burgess, T.F., Shaw, N.E. (2010), “Editorial Board Membership of Management and Business Journals: A Social Network Analysis Study of the Financial Times 40", British Journal of Management, 21: 627-648.

Butera F., Thurman, J.E. (1984), Automation and Work Design, Amsterdam, North-Holland.

Chabowski, B.R., Samiee, S., Hult, G. T. M. (2013), “A Bibliometric Analysis of the Global Branding Literature and a Research Agenda", Journal of International Business Studies, 44: 622-634.

Cheng, C.H., Kumar, A., Motwani, J.G., Reisman, A., Madan, M.S. (1999), “A Citation Analysis of the Technology Innovation Management Journals", IEEE Transactions on Engineering Management, 46: 4-13.

Chiesa, V., Coughlan, P., Voss, C.A. (1996), "Development of a technical innovation audit", Journal of Product Innovation Management, 13: 105-136. 
Christensen, C.M., Bower, J.L. (1996), "Customer power, strategic investment, and the failure of leading firms", Strategic Management Journal, 17: 197218.

Cook, S.D.N., Brown, J.S. (1999), "Bridging Epistemologies: The Generative Dance between Organizational Knowledge and Organizational knowing”, Organization Science, 10: 381-400.

Crossan, M. M., Apaydin, M. (2010), “A Multi-Dimensional Framework of Organizational Innovation: A Systematic Review of the Literature", Journal of Management Studies, 47: 1154-1191.

Davies, C., Dawson, S., Francis, A. (1973), “Technology and Other Variables: Some Current Approaches in Organization Theory", in Warner, M. (ed.), The Sociology of the Workplace: An Interdisciplinary Approach, New York, Wiley.

De Massis, A., Frattini, F., Lichtenthaler, U. (2013), "Research on Technological Innovation in Family Firms: Present Debates and Future Directions", Family Business Review, 26: 10-31.

Dewett, T., Jones, G.R. (2001), “The Role of Information Technology in the Organization: A Review, Model, and Assessment", Journal of Management, 27: 313-346.

Galende, J. (2006), “Analysis of Technological Innovation from Business Economics and Management", Technovation, 26: 300-311.

Garud, R., Tuertscher, P., Van de Ven, A.H. (2013), "Perspectives on Innovation Processes", Academy of Management Annals, 7: 775-819.

Hage, J. T. (1999), “Organizational Innovation and Organizational Change”, Annual Review of Sociology, 25: 597-622.

Hage, J., Aiken, M. (1969), "Routine Technology, Social Structure and Organizational Goals", Administrative Science Quarterly, 14: 366-376.

Harris, M. (2006), "Technology, innovation and post-bureaucracy: the case of the British Library", Journal of Organizational Change Management, 19: 80-92.

Jacobs, J.A. (2011), "Journal Rankings in Sociology: Using the H Index with Google Scholar", PSC Working Paper Series, PSC 11-05. http://repository.upenn.edu/psc_working_papers/29.

Katzy, B.R., Crowstonb, K. (2008), “Competency rallying for technical innovation-The case of the Virtuelle Fabrik", Technovation, 28(10): 679-692.

Knoben, J., Oerlemans, L. A. G., (2006), "Proximity and Inter-organizational Collaboration: A Literature Review", International Journal of Management Reviews, 8: 71-89. 
Kristof, A. L. (1996), "Person-organization fit: An integrative review of its conceptualizations, measurement, and implications", Personnel Psychology, 49: 1-49.

Leydesdorff, L. (2001), The Challenge of Scientometrics: The Development, Measurement, and Selforganization of Scientific Communications, Parkland, Universal Publications.

Liker, J. (1996), "Results of Survey of Management Journals for TIM Research", TIM Newsletter, 7: 5-8.

Linton, J.D., Thongpapanl, N. (2004), "Perspective: Ranking the Technology Innovation Management Journals", Journal of Product Innovation Management, 21: 123-139.

Lloréns Montes, F. J., Ruiz Moreno, A., García Morales, V. (2005), “Influence of support leadership and teamwork cohesion on organizational learning, innovation and performance: an empirical examination", Technovation, 25: 1159-1172.

Mansell, R., Avgerou, C., Quah, D., Silverstone, R. (2007), The Oxford Handbook of Information and Communication Technologies, Oxford, Oxford University Press.

Meissner, M. (1970), Technology and the Worker: Technical Demands and Social Processes in Industry, San Francisco, Chandler.

Nicolaisen, J. (2007), "Citation Analysis", Annual Review of Information Science and Technology, 41: 609-641.

Nieto, M. (2003), "From R\&D Management to Knowledge Management. An Overview of Studies of Innovation Management", Technological Forecasting \& Social Change, 70: 135-161.

Orlikowski, W.J. (2000), "Using Technology and Constituting Structures: A Practice Lens for Studying Technology in Organizations”, Organization Science, 11: 404-428.

Orlikowski, W.J., Scott S.V. (2008), "Sociomateriality: Challenging the Separation of Technology, Work and Organization", Academy of Management Annals, 2: 433-474.

Prajogo, D. I., Ahmed, P. K. (2006), "Relationships between Innovation Stimulus, Innovation Capacity, and Innovation Performance", $R \& D$ Management, 36: 499-515.

Santa, R., Bretherton, P., Ferrer, M., Soosay, C., Hyland, P. (2011), "The role of cross-functional teams on the alignment between technology innovation effectiveness and operational effectiveness", International Journal of Technology Management, 55: 122-137. 
Schumpeter, J. A. (1934), The Theory of Economic Development, Cambridge, MA, Harvard University Press.

Schumpeter, J. A. (1942), Capitalism, Socialism, and Democracy, New York, Harper \& Brothers.

Sen, F. K., Egelhoff, W. G. (2000), "Innovative capabilities of a firm and the use of technical alliances", Engineering Management, 47: 174-183.

Shane, S. (2000), "Prior Knowledge and the Discovery of Entrepreneurial Opportunities", Organization Science, 11: 448-469.

Sirilli, G., Evangelista, R., (1998), "Technological innovation in services and manufacturing: results from Italian surveys", Research Policy, 27: 881-899.

Small, H. (1973), "Co-citation in Scientific Literature: A New Measure of the Relationship between Two Documents", Journal of the American Society for Information Science, 24: 265-269.

Staropoli, C. (1998), "Cooperation in R\&D in the pharmaceutical industry - the network as an organizational innovation governing technological innovation", Technovation, 18: 13-23.

Teece, D.J. (1986), "Profiting from technological innovation: Implications for integration, collaboration, licensing and public policy", Research Policy, 15: 285-305.

Thongpapanl, N. (2012), "The Changing Landscape of Technology and Innovation Management: An Updated Ranking of Journals in the Field", Technovation, 32: 257-271.

Tushman, M.L., Anderson, P.C., O'Reilly, C. (1997), “Technology Cycles, Innovation Streams, and Ambidextrous Organizations: Organizational Renewal through Innovation Streams and Strategic Change", in Tushman, M. L., Anderson, P. C. (eds), Managing Strategic Innovation and Change, New York, Oxford University Press.

Van de Ven, A. H., Polley, D. (1992), "Learning while innovating", Organization Science, 3: 92-116.

Vogel, R. (2012), "The Visible Colleges of Management and Organization Studies: A Bibliometric Analysis of Academic Journals", Organization Studies, 33: $1015-1043$.

White, H.W., Wellman, B., Nazer N. (2004), "Does Citation Reflect Social Structure? Longitudinal Evidence from the "Globenet" Interdisciplinary Research Group", Journal of the American Society for Information Science and Technology, 55: 111-126.

Williamson, J. M., Lounsbury, J.W. Lee D.H. (2013), "Key personality traits of engineers for innovation and technology development", Journal of Engineering and Technology Management, 30: 157-168. 
Wingreen, S.C., Blanton, J.L. (2007), “A social cognitive interpretation of person-organization fitting: the maintenance and development of professional technical competency", Human Resource Management, 46: 631-650.

Wu, D.D., Kefan, X., Hua, L., Shi, Z., Olson, D. L. (2010), "Modeling technological innovation risks of an entrepreneurial team using system dynamics: an agent-based perspective", Technological Forecasting and Social Change, 77: 857-869.

Za, S., Spagnoletti, P. (2013), "Knowledge Creation Processes in Information Systems and Management: Lessons from Simulation Studies", in Spagnoletti, P. (ed.), Organizational Change and Information Systems, Lecture Notes in Information Systems and Organisation 2.

Zammuto, R.F., Griffith, T.L., Majchrzak, A., Dougherty, D.J., Faraj, S. (2007), "Information Technology and the Changing Fabric of Organization", Organization Science, 18: 749-762. 